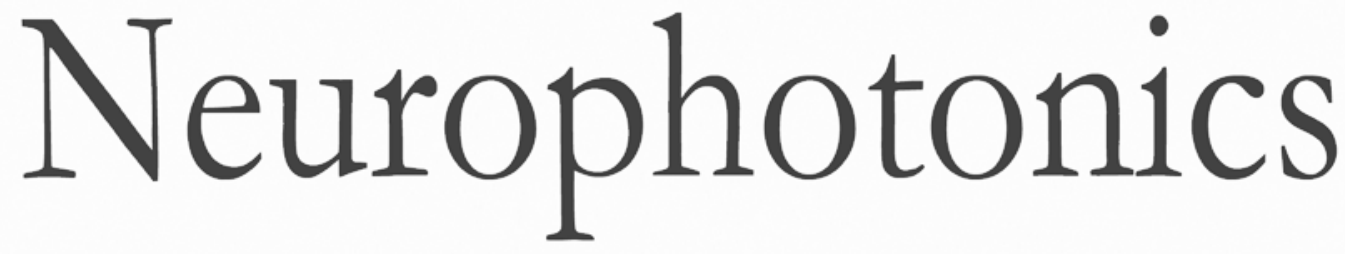

\title{
Prominent lateral spread of imaged evoked activity beyond cortical columns in barrel cortex provides foundation for coding whisker identity
}

Nathan S. Jacobs

Ron D. Frostig 


\title{
Prominent lateral spread of imaged evoked activity beyond cortical columns in barrel cortex provides foundation for coding whisker identity
}

\author{
Nathan S. Jacobs ${ }^{a, b}$ and Ron D. Frostig ${ }^{a, b, c, d, *}$ \\ ${ }^{a}$ University of California, Department of Neurobiology and Behavior, Irvine, California, United States \\ bUniversity of California, Center for Learning and Memory, Irvine, California, United States \\ 'University of California, Department of Biomedical Engineering, Irvine, California, United States \\ dUniversity of California, Center for Hearing Research, Irvine, California, United States
}

\begin{abstract}
The posterior medial barrel subfield (PMBSF) of a rat primary somatosensory cortex exquisitely demonstrates topography and columnar organization, defining features of sensory cortices in the mammalian brain. Optical imaging and neuronal recordings in rat PMBSF demonstrate how evoked cortical activity following single whisker stimulation also rapidly spreads laterally into surrounding cortices, disregarding columnar and modality boundaries. The current study quantifies the spatial prominence of such lateral activity spreads by demonstrating that functional connectivity between laterally spaced cortical locations is actually stronger than between vertically spaced cortical locations. Further, the total amount of evoked activity within and beyond single column boundaries was quantified based on intrinsic signal optical imaging, single units and local field potentials recordings, revealing that the vast majority of whisker evoked activity in PMBSF occurs beyond columnar boundaries. Finally, a simple two-layer artificial neural network model of PMBSF demonstrates the capacity of extracolumnar evoked activity spread to provide a foundation for accurate whisker stimulus classification that is robust to random scaling of inputs and local noise. Indeed, classification performance improved when more of the lateral spread was included in the model, providing further evidence for the relevance of the lateral spread. $\odot 2017$ Society of Photo-Optical Instrumentation Engineers (SPIE) [DOI: 10.1117/1.NPh.4.3.031218]
\end{abstract}

Keywords: point spread; posterior medial barrel subfield; rat; cortical columns; population code.

Paper 17011SSR received Jan. 17, 2017; accepted for publication May 12, 2017; published online Jun. 8, 2017.

\section{Introduction}

The rat primary somatosensory cortex, especially the posterior medial barrel subfield (PMBSF) representing the large, moveable whiskers (vibrissae), exquisitely demonstrates topography, a defining feature of sensory cortices in the mammalian brain. Each large whisker found on the rodent snout corresponds to a unique cytoarchitectural unit in layer IV of PMBSF known as the whisker barrel. Topographic organization is further supported by columnar organization in which neurons above, below, and within a barrel in layer IV respond preferentially to the same whisker. Topographic and columnar organization of PMBSF is supported by vertically oriented excitatory connections within barrel columns. ${ }^{1}$ PMBSF thus adheres to clear topographic and columnar principles of organization.

In addition to columnar and topographic boundaries, PMBSF also exhibits lateral spread of activity with prominent spatial footprints that span several $\mathrm{mm}$ in rats. Spatially broad profiles of activity evoked by "point" stimuli such as a single whisker are a ubiquitous and notable feature of sensory cortex ${ }^{2-20}$ (reviewed in Ref. 20). In PMBSF, lateral spread of activity is supported by an underlying plexus of horizontal intracortical projection fibers. ${ }^{14,21-23}$

The functional contributions of lateral spread in PMBSF have only recently begun to be explored..$^{20}$ The lateral spread has been linked to integration of cortical responses following multiwhisker stimulation ${ }^{16}$ and support whisker representations that are independent of absolute response magnitude and invariant to large changes in stimulus amplitude. ${ }^{19}$ Importantly, both studies found a primary role for whisker-evoked activity beyond cortical columns, rather than peak responses within columnar boundaries. These beyond-the-column findings were surprising as it is commonly assumed that only the strongest (or peak) response is the important variable for cortical processing and function. These findings, therefore, warrant a further study into the spread of cortical activity laterally beyond the column. The current study quantifies the prominence of the lateral spread beyond columnar boundaries using a functional connectivity analysis and cumulative response calculations. In addition, a simple artificial neural network model was used to demonstrate how activity patterns produced by lateral spread can support robust whisker identity coding. These findings provide further evidence for the importance of lateral spread beyond peak activity within PMBSF.

\section{Materials and Methods}

\subsection{Subjects}

All in vivo procedures were in compliance with the National Institutes of Health Guidelines and reviewed and approved by the University of California Irvine Animal Care and Use Committee. Subjects were adult male Sprague-Dawley rats. Rats were inducted with a bolus intraperitoneal injection of sodium pentobarbital (55 mg/kg b.w.) and maintained with 
supplemental injections as needed throughout the day. For intrinsic signal optical imaging (ISOI) data, 37 rats were used. For multidepth electrophysiology recordings, a different set of seven rats were used.

\subsection{Whisker Stimulation}

Whisker stimulation was restricted only to the right snout. Single whisker stimulation targeted the C2 whisker located centrally on the whisker pad [Fig. 3(a)]. As in previously established protocols, the stimulation of only whisker C2 was achieved with a copper wire probe attached to a computercontrolled stepping motor. Five deflections were delivered at $5 \mathrm{~Hz}$ rate for total time span of $1 \mathrm{~s}$. Each deflection displaced whisker $\mathrm{C} 2 \sim 1 \mathrm{~mm}$ along the rostrocaudal direction at an approximate speed of $0.25 \mathrm{~mm} / \mathrm{ms}$ (whisker probe position stabilized within $10 \mathrm{~ms}$ of initiation of movement) at a distance of $\sim 5 \mathrm{~mm}$ from the skin.

\subsection{Intrinsic Signal Optical Imaging}

ISOI was used for high-spatial resolution, wide field-of-view mapping of the total cortical activation spread evoked by whisker stimulation. Imaging was conducted with a 16-bit CCD camera (Cascade 512B II; Photometrics, Tucson, Arizona) combined with an inverted $50 \mathrm{~mm}$ lens plus extenders. The camera's field-of-view was a $7.42 \times 7.42 \mathrm{~mm}$ cortical region, mapped onto a $256 \times 256$ pixel array. For future alignment of data files collected within the same rats as well as across rats, the field-of-view neuroaxis was oriented the same in every rat, plus the field-of-view remained constant across data files within each rat. The CCD camera was focused $600 \mu \mathrm{m}$ below the cortical surface before the start of data collection to minimize contributions from surface blood vessels and maximize contributions integrated across the upper cortical layers. The imaged cortical region was continuously illuminated with a red LED (635 nm max, $15 \mathrm{~nm}$ full-width at half-height). Imaging frames were captured at $10 \mathrm{~Hz}$ rate (i.e., 1 frame $=100 \mathrm{~ms}$ exposure time), and each imaging trial lasted $15 \mathrm{~s}$. The onset of whisker stimulation occurred $1.5 \mathrm{~s}$ into the trial. A block of 64 trials was collected per whisker stimulation condition, with an intertrial interval averaging $6 \mathrm{~s}$ and ranging randomly between 1 and $11 \mathrm{~s}$ and thus an average of $21 \mathrm{~s}$ between the onset of consecutive stimulus deliveries. The 64 trials in a block were then summed and the summed data collapsed into 500-ms frames (referred to hereafter as a data file) to increase the signal-tonoise. For each data file, activity for each 500-ms poststimulus frame was converted to fractional change relative to the 500-ms frame collected immediately prior to stimulus onset on a pixelby-pixel basis. The current data set focused on "initial dip" frames in which the first local minimum of the ISOI signal occurs. C2 whisker barrel column and PMBSF boundaries were estimated using a representative map of whisker barrels derived from cytochrome-oxidase-stained sections.

\subsection{Electrophysiology}

Multisite, extracellular recordings were acquired using 32-channel arrays with an $8 \times 4$ design consisting of eight recording locations, each of which had four depths targeting layers 1 , 2/3, 4, and 5 [Fig. 2(a)]. Microelectrode arrays were made from insulated $35 \mu \mathrm{m}$ tungsten wire (California Fine Wire, Grover Beach, California) that were blunt cut and threaded in

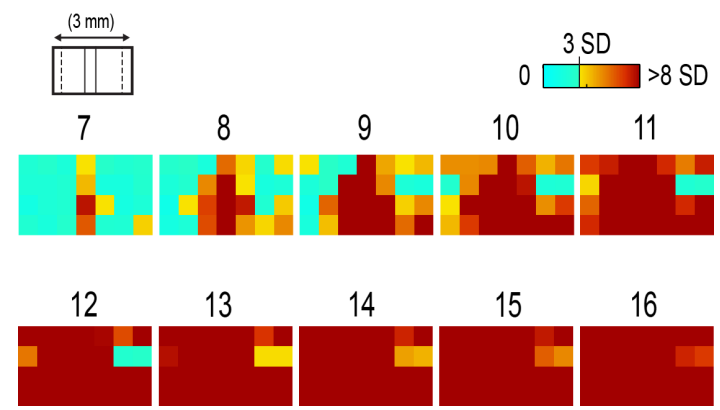

Fig. 1 Beyond columnar activity. Time series of a single whisker (C2) evoked LFP plotted as $z$-scores of baseline signal (mean, $n=6$, data set from Ref. 19, see Sec. 2 for details). Multisite recordings were taken from a planar section of rat PMBSF penetrating through the depth of cortex. Each frame consists of $7 \times 4$ recording locations in a multielectrode recording array sampling from cortical layer 1 (top of image), 2/3, 4, and upper layer 5 (bottom of image). Laterally, the array extends from just beyond PMBSF rostrally (left edge of image) to just beyond PMBSF caudally (right edge of image). Initial evoked activity is seen across vertical column of tissue consistent with traditional columnar boundaries. This brief $(1 \mathrm{~ms})$ initial pattern is rapidly transformed to extensive lateral spread of cortical responses into surround cortex. Within milliseconds, evoked activity $>3 \mathrm{SD}$ above prestimulus noise is seen across all sampling locations. Inset, upper left, simplified schematic of electrode array positions [for detail see (a)]. Dark lines denote columnar boundaries of C2 whisker barrel, dotted lines indicate PMBSF boundaries.

groups of four through polyimide guide tubes spaced $0.5 \mathrm{~mm}$ apart. Mean impedance of electrodes was $153 \mathrm{k} \Omega \pm 55$ (measured with IMP-2, Bak electronics, Sanford, Florida). Raw signals starting $1 \mathrm{~s}$ before and ending $1 \mathrm{~s}$ after stimulus onset (total of $3 \mathrm{~s}$ per trial) were amplified and digitized at a 22-kHz sample rate (SnR system, Alpha Omega, Nazareth, Israel). The distance to $\mathrm{C} 2$ whisker barrel was measured in cytochrome oxidase stained tissue sections. For PSTHs, 1-ms time bins were used.

Postprocessing was done using custom MATLAB ${ }^{\circledR}$ scripts. Raw traces were band-pass filtered for local field potentials (LFP, 1 to $300 \mathrm{~Hz}$ ) or spikes (300 to $3 \mathrm{k} \mathrm{Hz}$ ) using a twopole Butterworth function. Trials with electrical noise $(5.32 \%$ of trials) were excluded from trial averages. For the few bad channels in arrays $(5.36 \%$ of channels overall, equivalent to 1.6 channels per array), trial averages from adjacent channels at the same cortical depth were averaged. In trial averaged LFP, mean baseline values 50 to $0 \mathrm{~ms}$ before stimulus onset were subtracted. A Gaussian filter was used to remove electrical noise near $60 \mathrm{~Hz}$. Data were downsampled to a $10-\mathrm{kHz}$ sample rate for further analysis.

For $z$-normalized LFP data in Fig. 1, responses were divided by the standard deviation of signal in the $10-\mathrm{ms}$ time period before whisker stimulation on a trial-by-trial basis. This resulted in values that indicated the number of standard deviations from baseline noise a given response was.

\subsection{Whisker Identity Coding Model Based on Spatial Pattern of Activity}

Whisker coding based on mesoscopic patterns of evoked activity was assessed using a two-layer artificial neural network consisting of a $5 \times 5$ input layer ("PMBSF" with 25 "whisker barrels") and a single output unit (downstream "reader" neuron).

The network architecture was similar to a perceptron. ${ }^{24}$ Input units were only connected to the output unit. There was no 
feedback onto the input layer. Synaptic weights were randomly set to a value between -1 and 1 . The degree and arrangement of connectivity between the input and output layers was varied to produce four network types [see Fig. 4(c)]: (1) single connection between input layer and output unit, (2) four adjacent units of input layer connected to output unit, (3) four maximally spaced units of input layer connected to output unit, and (4) all units of input layer connected to output unit. This set of network architectures was chosen because it included variations in the degree of connectivity ( 1 versus 4 versus 25 inputs) as well as variations in the heterogeneity of connections (adjacent versus spaced inputs).

Each trial consisted of two time steps: (1) activation of input layer and (2) activation of output unit. Input activity was set by empirically derived activity patterns (see below) with values ranging between 0 and 1 . Output unit activity was binary ( 0 or 1$)$ and calculated using a thresholded, linear activation function with no bias constant and threshold $=0$.

Input activity patterns were empirically derived from previously published ISOI data. ${ }^{16}$ A response decay rate function was derived from average whisker C2-evoked ISOI "initial dip" data obtained by averaging results from 37 rats [Fig. 4(a), left panel]. Four linear sections starting at the peak response and extending radially outward each separated by 90 deg were averaged, peaknormalized, and fitted with a two component exponential function [Fig. 4(a), middle panel]: $0.2 \times \exp [-0.07 \times(x \times s)]+$ $0.83 \times \exp [-0.014 \times(x \times s)]$, where $s=21$ to convert from spatial scale of empirical data $(1 \mathrm{pixel}=28 \mu \mathrm{m})$ to spatial scale of model input layer ( 1 pixel $=0.5 \mathrm{~mm}$, approximate distance between barrels in rat PMBSF). The decay rate function was used to produce 25 different activity patterns with peak activation over each of the 25 pixels of the $5 \times 5$ input layer [Fig. 4(a), right panel]. Lastly, in order to model changes in stimulus intensity, on every trial the activity pattern was also uniformly scaled by a random factor between 0 and 1 pulled from a uniform distribution function $[\operatorname{rand}()$ function in MATLAB ${ }^{\circledR}$ ]. Uniform scaling of mesoscopic response patterns in PMBSF across a wide range of stimulus intensity has previously been shown. ${ }^{19}$

Each trial was randomly selected to either include presentation of the target input $(\mathrm{C} 3$, with peak activation over the "barrel" at the very center of the $5 \times 5$ input layer) or an offtarget input (all other whisker inputs). For trials of off-target inputs, one of the 24 nontarget input activity patterns was randomly selected. Thus, half of all trials included the target input, and chance performance had 50\% accuracy. Performance ( $\%$ of trials accurately classified as on or off target) was assessed in blocks of 100 trials called a "session." Training consisted of 20 sessions (total of 2000 trials) and testing consisted of 10 sessions (total of 1000 trials). Synaptic weights were updated during training (trials 1 to 2000) using the delta rule: $\Delta \omega_{i j}=\alpha \times\left(t_{j}-y_{j}\right) \times x_{i}$, where $\omega$ is synaptic weight, $i$ is the presynaptic unit, $j$ is the postsynaptic unit, $\alpha$ is the learning rate constant, $t$ is the target response, $y$ is the actual response, and $x$ is the unit activity. The simulation was repeated for 100 networks (per network type).

Network simulations were repeated for three conditions: (1) no noise, (2) noise, and (3) noise with complex classification task. The no noise condition is described above. For the noise condition, independent noise (random value between -0.1 and 0.1 ) was injected into each unit of the input layer on each trial. For comparison, peak values in input patterns ranged between 0 and 1 with a theoretical mean of 0.5 . Thus, the maximum signal-to-noise ratio for peak values under these conditions was 10 , with a theoretical average of 5 , and even lower signalto-noise ratios for all off-peak values.

For the last condition, noise was again added and the target input was made more challenging-the output unit had to respond to both the $\mathrm{C} 3$ and A5 inputs (while still correctly rejecting all other inputs). MATLAB ${ }^{\circledR}$ source code at can be found in Ref. 25.

\subsection{Statistics}

Cross correlations, modeling, and descriptive statistics were performed using custom MATLAB ${ }^{\circledR}$ scripts. All parametric statistics were performed in SYSTAT version 11.

For cumulative response magnitudes, responses between 0 and $0.22 \mathrm{~mm}, 0.5,1.0$, or $1.5 \mathrm{~mm}$ from location of peak activity were integrated. For LFP and multiunit (MU) data, response value at $0.22 \mathrm{~mm}$ was interpolated (linear interpolation) from response values at 0 and $0.5 \mathrm{~mm}$ and the trapezoid method of integration used for cumulative response calculations. (a)

$$
\text { Multisite recordings }
$$

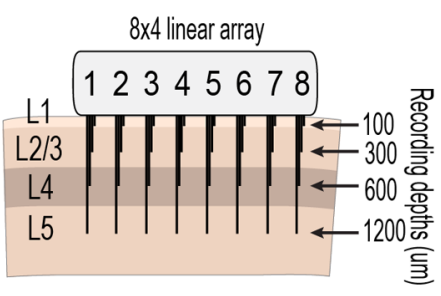

(b)

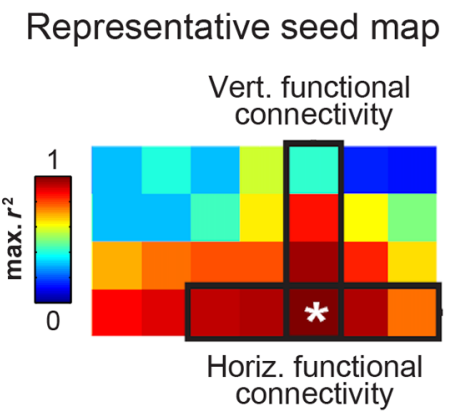

(c)

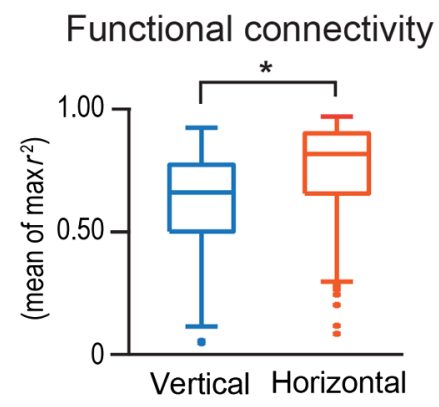

Fig. 2 Horizontal and vertical functional connectivity in the barrel cortex. (a) Multielectrode array design to sample from most cortical layers (up to $1.2 \mathrm{~mm}$ deep) and across the full breadth of rat barrel cortex (3.5 mm across). (b) Cross correlations on C2 evoked LFP data from Ref. 19 were run for each recording location. Mean $r^{2}$ values within nearby horizontal recording locations were interpreted as "horizontal" functional connectivity. Mean $r^{2}$ values for three other electrodes within the bundle were interpreted as "vertical" functional connectivity. (c) Group statistics show stronger functional connectivity along the horizontal axis (within layer) as compared to the vertical axis (within column) for single whisker stimulation $(\mathrm{C})$. 
Cross-correlations of evoked LFP with $\pm 50 \mathrm{~ms}$ lag times were run between pairs of recording locations of an $8 \times 4$ array of electrodes using MATLAB's xcorr() function. Each recording location was used as a seed and cross correlations run with locations within the same vertical column (for vertical connectivity measure) or within the same horizontal row (for horizontal connectivity measure).

For box and whisker plots in Fig. 2, solid line indicates the median, outer bounds of box indicate upper and lower quartiles, whiskers show minimum and maximum values (excluding outliers), and dots represent outliers using the following default formula for box plots in MATLAB ${ }^{\circledR}$ : maximum whisker length $w$ is set to 1.5 times the interquartile range. Outliers are any data points greater than $q 3+w(q 3-q 1)$ or smaller than $q 1-w(q 3-q 1)$, where $q 1$ and $q 3$ are the 25th and 75th percentiles, respectively.

\section{Results}

\subsection{Lateral Spread of Whisker Evoked Cortical Activity}

The stimulation of a single whisker in rats evokes a prominent lateral spread of activity through PMBSF and into surrounding cortices. An example of a single whisker evoked LFP in PMBSF is shown in Fig. 1, which shows response magnitudes in standard deviations from baseline noise (see Sec. 2 for details). Every sampling location-which included electrodes from four different cortical layers extending beyond the boundaries of PMBSF-recorded evoked LFP that was over three standard deviations above baseline noise. These data suggest that evoked activity is only constrained to a cortical column for $1 \mathrm{~ms}$ following single whisker stimulation, before spreading laterally beyond columnar boundaries and engaging a broad volume of cortex.

Robust horizontal functional connectivity would be consistent with the prominent lateral spread of evoked activity; however, this has not been explicitly tested before. Therefore, functional connectivity along vertical and horizontal domains in PMBSF was compared using cross-correlations of multisite recordings of a single whisker (C2) evoked LFP (Fig. 2). Correlation coefficients for cross-correlations with a seed location were binned vertically or horizontally [see representative seed map in Fig. 2(b)] and then averaged for all seed locations. Mean peak $r^{2}$ values were significantly higher for horizontal connectivity compared to vertical connectivity $[p=0.001$ (a) Stimulation

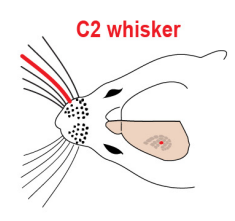

(b) Single whisker evoked ISOI

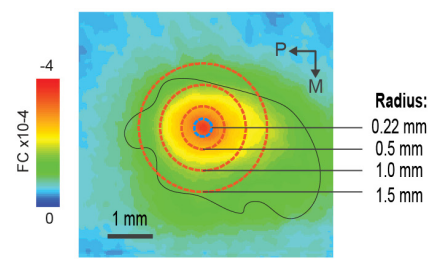

(e)

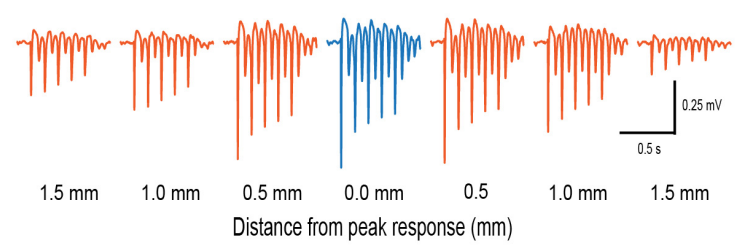

(h)

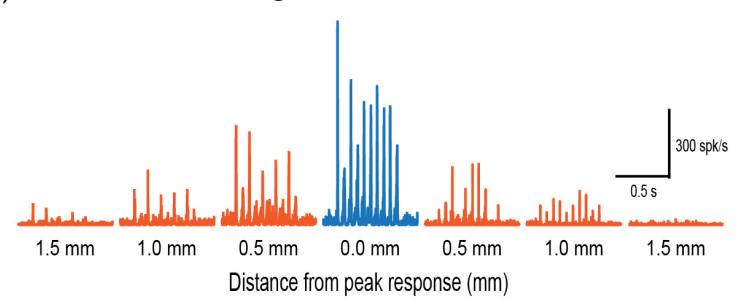

(c)

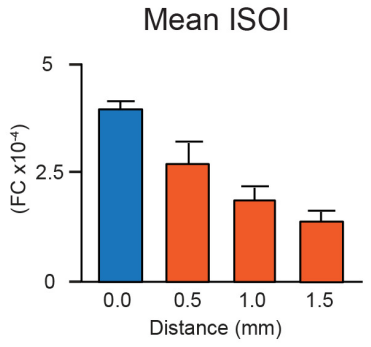

(f)

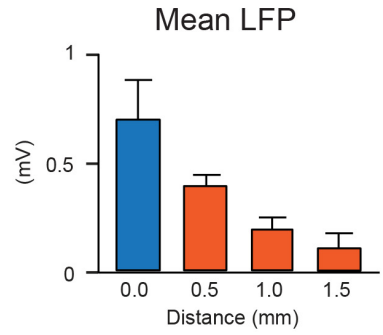

(i)

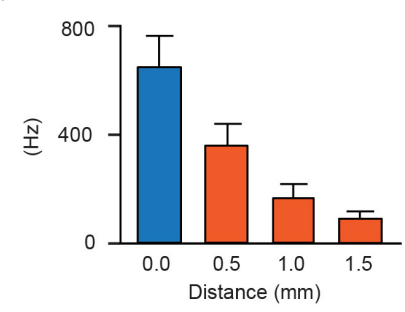

(d)

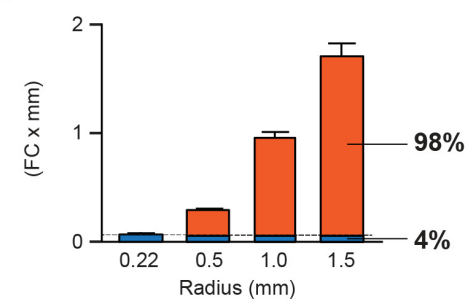

(g)

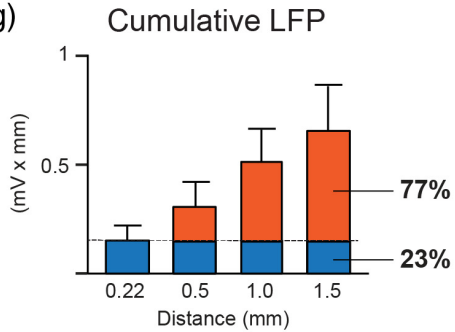

(j)

Cumulative MU

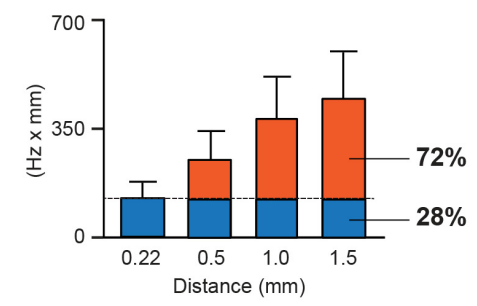

Fig. 3 Single whisker evokes more activity outside the barrel column. (a) Schematic of single whisker and anatomical representation in PMBSF. (b, e, h) Single whisker evoked activity in PMBSF measured by ISOI "initial dip" signal (b, data set from Ref. 16), LFP (e, data set from Ref. 19), and multiunit activity (h, data set from Ref. 19). FC, fractional change. (c, f, i) Mean response magnitudes at 0, 0.5, 1 , and $1.5 \mathrm{~mm}$ from location of peak c2 evoked response for (c) ISOI, (f) LFP, and (i) MU activity. (d, g, j) Cumulative responses at $0.22,0.5,1.0$, and $1.5 \mathrm{~mm}$ from location of peak $\mathrm{C} 2$ evoked response for (d) ISOI, (g) LFP, and (j) MU activity. (d) For two-dimensional ISOI data, responses were integrated within specified radius. $(\mathrm{g}$, j) For one-dimensional electrophysiology data, responses were integrated between $0 \mathrm{~mm}$ and specified distance from peak response. Responses at $0.22 \mathrm{~mm}$ were estimated using linear interpolation. $(\mathrm{e}, \mathrm{h})$ Representative single whisker evoked LFP (e) and multiunit PSTHs (h) at increasing distances from peak response. Recording depth $=600 \mu \mathrm{m}$. Solid black line in b denotes PMBSF boundary. 
(a)

Generalized PMBSF mesoscopic activity profile
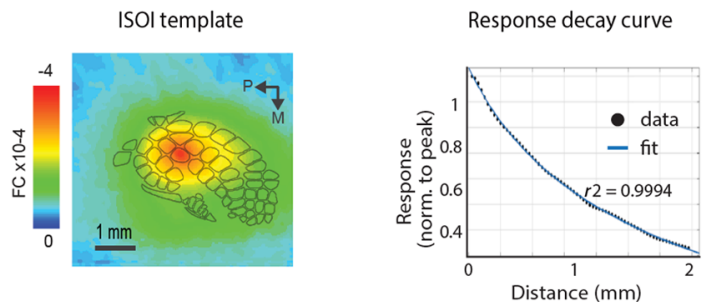

Simplified spatial pattern of activity

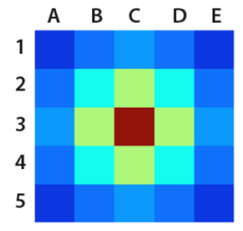

(b)

Variable stimulus intensity ambiguates whisker coding
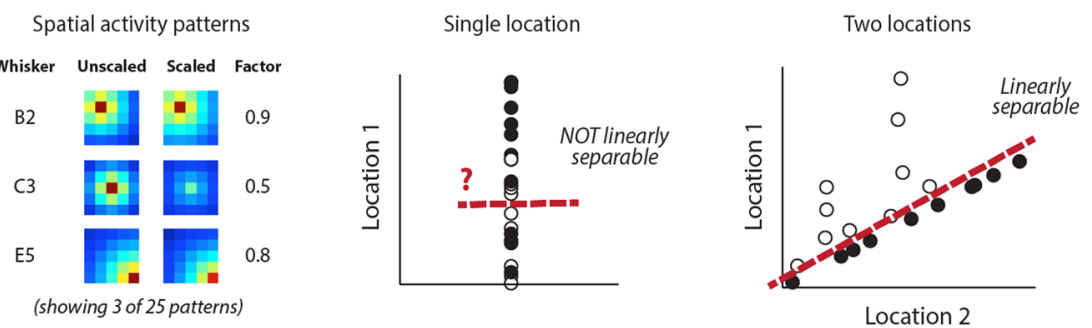

(c)

Network architectures for downstream "readers"
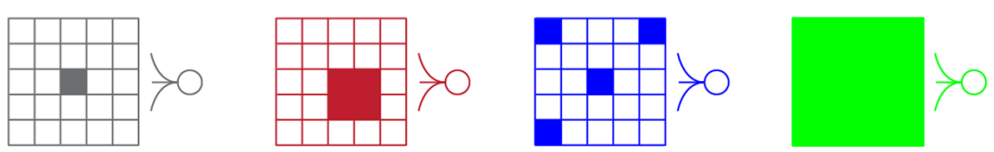

(d)

Robust whisker coding with mesoscopic activity
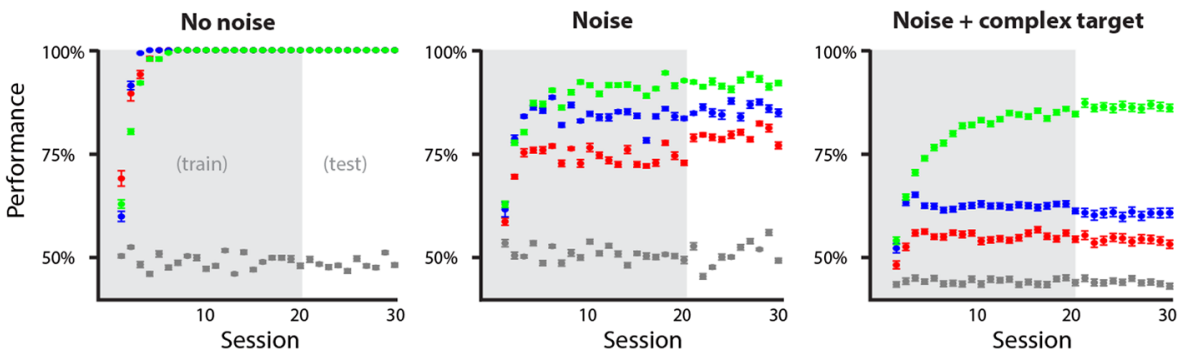

Fig. 4. Spatial patterns of activity support robust whisker identity coding scheme. A simple two-layer neural network model was used to test the robustness of whisker coding based on large spatial patterns of cortical activity. (a) Simplified cortical activity patterns were derived from mean whisker C2-evoked ISOI "initial dip" data from rat PMBSF ( $n=37$, data set from Ref. 16). Left panel, mean ISOI "initial dip" data used to fit the decay rate function. Middle panel, ISOI response magnitudes plotted against cortical distance from peak response (black circles) with overlay of fitted response decay function (blue line). Right panel, example of a $5 \times 5$ stimulus pattern $(1$ pixel $=0.5 \mathrm{~mm}$ ) generated by the response decay function. Scale bar, left panel $=1 \mathrm{~mm}$. (b) Before being used as inputs into the model, stimuli were multiplied by a randomized scalar to capture the variable intensity of whisker stimuli (left panel). Each whisker stimulus would therefore vary greatly in absolute response magnitude from trial to trial while the relative pattern of activity remained exactly the same, consistent with a previous report of invariant relative spatiotemporal profile of activity across large ranges of stimulus intensities. ${ }^{19}$ Given the slow response decay rate, these conditions made it effectively impossible to classify whisker stimulus identity (e.g., whisker C3) using responses at any one location (middle panel). If responses from two locations are considered, the classification problem is greatly simplified (right panel). (c) Gray, reader unit connected to single location of input layer located at the peak response for the target stimulus (C3). Red, reader unit connected to four adjacent units of the input layer all within close proximity to the peak response for target stimulus. Blue, reader unit connected to four units of the input layer, one at the location of peak response for target stimulus and three additional units at maximal distances from the first. Green, reader unit connected to all units of the input layer. (d) Whisker classification performance for each reader type. Classification accuracy for all readers was tested in three conditions of increasing difficulty: no noise (left panel), noise (middle panel), and noise with a complex target where readers had to respond to both the C3 whisker pattern as well as the A5 whisker pattern (right panel, "complex target"). Error bars = SEM. 
paired $t$-test; Fig. 2(c)]. These data suggest a robust horizontal connectivity that is stronger than the well-established vertical connectivity in PMBSF.

\subsection{Prominent Cumulative Evoked Activity Beyond Columnar Boundaries}

Stimulation of a single whisker in rats evokes responses within columns of cortical tissue in PMBSF that rapidly spreads laterally into surrounding cortices (Fig. 1). The cortical response to single whisker stimulation can thus be broken into two components: peak responses localized within cortical columns (strong response, small volume, Fig. 3, blue responses) and a broad profile of weaker responses (weak response, large volume, Fig. 3, orange responses). To determine whether most single whisker evoked activity in PMBSF occurs near peak responses (i.e., within columnar boundaries) or in surrounding cortices (i.e., beyond columnar boundaries), cumulative response magnitudes were calculated.

Following single whisker C2 stimulation [Fig. 3(a)], cumulative response magnitudes (response magnitude integrated across space) were calculated for increasing distances/areas from the location of peak responses for ISOI initial dip data [Fig. 3(d), $n=37$, data set from Ref. 16], LFP [Fig. 3(g), $n=6$, data set from Ref. 19], and multiunit activity [MU, Fig. 3(j), $n=6$, data set from Ref. 19]. A distance/radius of $0.22 \mathrm{~mm}$ was used as the standard demarcation for columnar boundaries based on the $0.15 \mathrm{~mm}^{2}$ area of the $\mathrm{C} 2$ whisker barrel. ${ }^{9}$ Cumulative response magnitudes were calculated up to a distance/radius of $1.5 \mathrm{~mm}$, roughly corresponding to the boundaries of PMBSF as measured from the $\mathrm{C} 2$ whisker barrel. On average, the cumulative response between 0 and $0.22 \mathrm{~mm}$ from peak responses (i.e., total activity within columnar boundaries) was only $4 \%, 23 \%$, and $28 \%$ of the cumulative response between 0 and $1.5 \mathrm{~mm}$ from peak responses (i.e., total activity within and beyond columnar boundaries, up to $1.5 \mathrm{~mm}$ ) for ISOI, LFP, and multiunit data sets, respectively. The lateral spread of evoked multiunit activity tends to be more spatially restricted than evoked $\mathrm{LFP}^{14}$ and ISOI activity, ${ }^{16}$ which could explain the range in cumulative response magnitudes beyond columnar boundaries. For both activity measures, these data show that the vast majority of whisker-evoked activity (as much as $98 \%$, for ISOI data) occurs beyond columnar boundaries. These percentages would be even smaller if the entire lateral spread of evoked activity, which extends beyond PMBSF boundaries, ${ }^{14}$ were included.

\subsection{Robust Whisker Identity Coding Using Evoked Activity Spread}

What is the purpose of all this extracolumnar activity? A possible role in providing a robust foundation for whisker coding is explored.

Robust coding of whisker identity with spatial activity patterns was tested using a two-layer artificial neural network model of PMBSF and a downstream "reader" neuron. The input layer of the network was a $5 \times 5$ grid representing a simplified model of PMBSF with 25 whisker barrels separated by $0.5 \mathrm{~mm}$ [Fig. 4(a), right panel]. The pattern of activity across the input layer was empirically derived from C2-evoked ISOI "initial dip" data, ${ }^{16}$ which was used to create a standard curve for the decay rate of single whisker evoked point spreads in rat PMBSF [Fig. 4(a), middle panel]. The simplified $5 \times 5$ patterns of activity were used to demonstrate an inherent ambiguity in whisker evoked columnar activity and, combined with downstream "reader" output units, to test whether spatial patterns of activity could support a robust whisker identity coding mechanism.

If input patterns are scaled by a random factor across trials [Fig. 4(b), left panel], there is no simple way of classifying whisker identity using input activity from just one location of the input layer [Fig. 4(b), middle panel]. This would be analogous to receiving afferents from neurons in a single cortical column. The difficulty in classifying whisker stimulus identity using activity from a single cortical location arises from the overlap in response magnitude distributions produced by a range of possible whisker stimulus intensities. For example, weak stimulation of the $\mathrm{C} 2$ whisker may produce a weaker response in the $\mathrm{C} 2$ barrel than strong stimulation of the $\mathrm{C} 3$ whisker. A simple way to resolve this inherent ambiguity is to integrate activity from multiple locations across PMBSF [Fig. 4(b), right panel].

Four network architectures with varying degrees of connectivity between the input layer and the output unit were investigated [Fig. 4(c)]. Classification performance of each network architecture was compared across three conditions of increasing difficulty—no noise [Fig. 4(d), left panel], noise [Fig. 4(d), bottom middle panel], and noise with a complex target [Fig. 4(d), right panel]. Results show that performance above chance (50\%) required readers to be connected to multiple units of the input layer [Fig. 4(d), compare gray circles to all other circles]. Performance improved if the reader was connected to more heterogeneous units of the input layer [Fig. 4(d), compare blue and red circles]. Performance was best if the reader was fully connected to the entire input layer [Fig. 4(d), compare green and blue circles]. These results show that using the full spatial profile of activity [Fig. 4(d), green network and circles] can support whisker coding that is robust to random scaling of input patterns, local noise designed to produce an average signalto-noise ratio of 5 for peak responses and 2.5 for more distal responses $(+1.5 \mathrm{~mm}$ from peak response, see Sec. 2 for details), and supports learning a classification scheme in which the output unit responds to either of two nonadjacent whiskers but not to any others [with $85 \%$ accuracy for fully connected output unit, Fig. 4(d), right panel, green circles].

In summary, these results suggest that stimulating a single whisker engages the entirety of PMBSF (Fig. 1) with stronger horizontal functional connectivity than vertical functional connectivity (Fig. 2), evokes more activity beyond columnar boundaries than within (Fig. 3), and produces spatial patterns of activity that support highly robust whisker identity coding (Fig. 4).

\section{Discussion}

Single whisker stimulation initially evoked activity within a cortical column ${ }^{26}$ that ignites an expansive profile of activity extending across and beyond PMBSF. ${ }^{14}$ This finding was demonstrated again here (Fig. 1) and further supported by a horizontal functional connectivity that was stronger than vertical functional connectivity (Fig. 2). Importantly, the current findings demonstrate that rather than being the minority response, weaker, off-peak responses beyond columnar boundaries actually constitute the large majority of whisker evoked activity (Fig. 3). This raises an important question: what is the functional role of the majority of whisker evoked activity in PMBSF? 
Lateral spread of evoked activity has been linked to two important sensory functions in PMBSF, multiwhisker integration $^{16}$ and invariance. ${ }^{19}$ The current findings suggest a possible third function of prominent lateral spread of evoked activitythe robust coding of whisker identity. To test this, a simple twolayer artificial neural network similar to the perceptron ${ }^{24}$ was used with spatially organized input patterns derived empirically from ISOI data [Fig. 4(a)]. Spatial patterns of activity across PMBSF resolve an inherent ambiguity introduced by variable stimulus intensity [Fig. 4(b)]. Increasing the degree and variety of sampling of input patterns improved the accuracy of whisker coding [Figs. 4(c) and 4(d)]. The current findings reinforce the notion that coding is optimal with large, heterogeneous neural populations ${ }^{27}$ and demonstrate that even highly simplified spatial activity patterns can support highly robust whisker coding. The capacity for classifying whisker input sources should also hold for multiwhisker stimuli, provided they evoke unique spatial patterns of activity.

It should be noted that it is well known that even simple neural network architectures such as the perceptron can exhibit robustness to noise ${ }^{28}$ and perform classification tasks. ${ }^{29}$ In the current study, these findings were simply replicated with a specific use case to demonstrate the ease with which whisker evoked activity patterns in PMBSF can be used to code for whisker stimulus identity.

The current study attempted to answer a simple question: Where does most whisker-evoked activity occur within PMBSF? Finding that the large majority of whisker evoked activity occurs beyond columnar boundaries (spatial prominence) raises a second question: what is the role of this extra columnar spatial prominence? In addition to previously discovered roles in multiwhisker integration ${ }^{16}$ and invariance, ${ }^{19}$ the current findings suggest that spatial patterns of activity may play a more general role as a foundation for robust whisker identity coding. Together, these findings demonstrate that spatially prominent, extracolumnar whisker evoked activity is a primary response feature in PMBSF.

If weaker off-peak lateral evoked responses are unimportant in PMBSF, why do they exist at all? And why should the cortex maintain a system of horizontal projections to support them, rather than supporting only columnar activation? The current study answers this question in two important ways. First, we show with three complimentary neurophysiology data sets that despite weaker absolute response magnitudes, extracolumnar activity actually constitutes the large majority of whiskerevoked activity in PMBSF. Second, we used a simple artificial neural network to show that spatial patterns of activity provide a highly robust foundation for whisker coding that is independent of absolute response magnitudes. Together the current results suggest that whisker evoked activity beyond columnar boundaries are as important for PMBSF as the columnar response. and therefore, the two should not be separated.

\section{Disclosures}

The authors have no financial conflicts of interest to report.

\section{Acknowledgments}

This work was supported by the NIH-NINDS NS-066001 and NS-055832, and the Center for Hearing Research NIH Training Grant 1T32DC010775-01.

\section{References}

1. D. Feldmeyer, "Excitatory neuronal connectivity in the barrel cortex," Front. Neuroanat. 6, 24 (2012).

2. H. S. Orbach, L. B. Cohen, and A. Grinvald, "Optical mapping of electrical activity in rat somatosensory and visual cortex," J. Neurosci. 5(7), 1886-1895 (1985).

3. A. Grinvald et al., "Functional architecture of cortex revealed by optical imaging of intrinsic signals," Nature 324, 361-364 (1986).

4. A. Grinvald, E. E. Lieke, and R. D. Frostig, "Cortical point-spread function and long-range lateral interactions revealed by real-time optical imaging of macaque monkey primary visual cortex," J. Neurosci. 14, 2545-2568 (1994).

5. D. S. Barth et al., "The spatiotemporal organization of auditory, visual, and auditory-visual evoked potentials in rat cortex," Brain Res. 678, 177-190 (1995).

6. A. Das and C. D. Gilbert, "Long-range horizontal connections and their role in cortical reorganization revealed by optical recording of cat primary visual cortex," Nature 375, 780-784 (1995).

7. J. S. Bakin et al., "Suprathreshold auditory cortex activation visualized by intrinsic signal optical imaging," Cereb. Cortex 6, 120-130 (1996).

8. V. Bringuier et al., "Horizontal propagation of visual activity in the synaptic integration field of area 17 neurons," Science 283, 695-699 (1999).

9. B. A. Brett-Green, C. H. Chen-Bee, and R. D. Frostig, "Comparing the functional representations of central and border whiskers in rat primary somatosensory cortex," J. Neurosci. 21, 9944-9954 (2001).

10. S. Kaur et al., "Spectral integration in primary auditory cortex: laminar processing of afferent input, in vivo and in vitro," Neuroscience 134, 1033-1045 (2005).

11. I. Ferezou et al., "Spatiotemporal dynamics of cortical sensorimotor integration in behaving mice," Neuron 56, 907-923 (2007).

12. I. Ferezou, S. Bolea, and C. C. Petersen, "Visualizing the cortical representation of whisker touch: voltage-sensitive dye imaging in freely moving mice," Neuron 50, 617-629 (2006).

13. D. Sharon et al., "Cortical response field dynamics in cat visual cortex," Cereb. Cortex 17, 2866-2877 (2007).

14. R. D. Frostig et al., "Large-scale organization of rat sensorimotor cortex based on a motif of large activation spreads," J. Neurosci. 28, 1327413284 (2008).

15. H. Adesnik and M. Scanziani, "Lateral competition for cortical space by layer-specific horizontal circuits," Nature 464(7292), 1155-1160 (2010).

16. C. H. Chen-Bee et al., "Whisker array functional representation in rat barrel cortex: transcendence of one-to-one topography and its underlying mechanism," Front. Neural Circuits 6, 93 (2012).

17. J. C. Wester and D. Contreras, "Columnar interactions determine horizontal propagation of recurrent network activity in neocortex," J. Neurosci. 32(16), 5454-5471 (2012).

18. M. H. Mohajerani et al., "Spontaneous cortical activity alternates between motifs defined by regional axonal projections," Nat. Neurosci. 16, 1426-1435 (2013).

19. N. S. Jacobs, C. H. Chen-Bee, and R. D. Frostig, "Emergence of spatiotemporal invariance in large neuronal ensembles in rat barrel cortex," Front. Neural Circuits 9, 34 (2015).

20. R. D. Frostig et al., "Imaging Cajal's 'neuronal avalanche': how widefield optical imaging of the point-spread advanced the understanding of neocortical structure-function relationship," Neurophotonics 4(3) (2017).

21. R. T. Narayanan et al., "Beyond columnar organization: cell type-and target layer-specific principles of horizontal axon projection patterns in rat vibrissal cortex," Cereb. Cortex 25(11), 4450-4468 (2015).

22. B. A. Johnson and R. D. Frostig, "Long, intrinsic horizontal axons radiating through and beyond rat barrel cortex have spatial distributions similar to horizontal spreads of activity evoked by whisker stimulation," Brain Struct. Funct. 221(7), 3617-3639 (2016).

23. J. Stehberg, P. T. Dang, and R. D. Frostig, "Unimodal primary sensory cortices are directly connected by long-range horizontal projections in the rat sensory cortex," Front. Neuroanat. 8, 93 (2014).

24. F. Rosenblatt, "The perceptron: a probabilistic model for information storage and organization in the brain," Psychol. Rev. 65(6), 386-408 (1958).

25. NS Jacobs, “Jacobs-2017: source code," 2017, https://zenodo.org/ badge/latestdoi/87992624. 
26. M. Armstrong-James, K. Fox, and A. Das-Gupta, "Flow of excitation within rat barrel cortex on striking a single vibrissa," J. Neurophysiol. 68(4), 1345-1358 (1992).

27. M. A. L. Nicolelis and M. A. Lebedev, "Principles of neural ensemble physiology underlying the operation of brain-machine interfaces," Nat. Rev. Neurosci. 10(7), 530-540 (2009).

28. R. Khardon and G. Wachman, "Noise tolerant variants of the perceptron algorithm," J. Mach. Learn. Res. 8, 227-248 (2007).

29. M. Opper and D. Haussler, "Generalization performance of Bayes optimal classification algorithm for learning a perceptron," Phys. Rev. Lett. 66(20), 2677-2680 (1991).
Nathan S. Jacobs has a PhD in neurobiology and behavior from UCI focusing on in vivo imaging and recording of population level activity in sensory cortex. He currently develops mobile applications for academic and research applications at UCLA.

Ron D. Frostig, PhD, is a professor at $\mathrm{UCl}$ with a research focus on the structure and function of the sensory cortex with special emphasis on plasticity (its ability to change its structure and function). We believe that plasticity is a fundamental property that underlies both normal cortical function (from sensory processing to learning and memory) and cortical recovery from deprivation and injury. 